\title{
Neutron Coincidence Measurements and Monte Carlo Modelling of Waste Drums Containing Reference Nuclear Material
}

\author{
A. Borella, R. Rossa, S. Boden, C. Bruggeman, B. Rogiers, S. Smets, E. Valcke \\ Belgian Nuclear Research Centre, Belgium \\ corresponding author: alessandro.borella@ sckcen.be
}

\begin{abstract}
Within the EC-funded CHANCE project several non-destructive techniques are being considered for the assay of waste bearing drums. Such techniques include calorimetry, gamma-ray spectrometry and neutron coincidence counting. The aim is to quantify uncertainties on the inventory of radionuclides, and how these are potentially reduced by combining the signatures from different techniques in the data analysis.
\end{abstract}

In this framework, neutron coincidence measurements were carried out with two slab counters based on ${ }^{3} \mathrm{He}$ detectors coupled to shift register electronics. Such a system consists of two identical slabs with 6 detectors each, and is transportable, rather compact and flexible in terms of sizes and geometries that can be measured. With this system three $200 \mathrm{~L}$ drums containing certified reference nuclear material and different filling materials were measured. The certified nuclear material was in the form of 21 pellets of mixed oxide of $U$ and Pu with a total mass of about $10.5 \mathrm{~g}$; in addition, a single pellet of about $10.05 \mathrm{~g}$ was also available. The pellets could be placed in predefined positions within the drum in a reproducible way. The geometry and composition of the three drums was well characterized and consisted of Ethafoam, a mixture of Ethafoam, stainless steel and PVC, and mortar with an inner core of extruded polystyrene. The measurement setup was arranged such that the drum was placed between the two slab counters. The positions of the slab counters relative to the drum were accurately measured before each measurement, and a dedicated system was used to minimize the uncertainty on the detector positioning.

The measurement data were first analysed by applying the point model of Hage and the mass of nuclear material in the drum was determined from the rate of totals and reals and the radionuclide composition. Due to the fact that not all the point model conditions were met, we found that the point model overestimates the mass up to about $50 \%$. In addition, a Monte Carlo model of the measurement geometry was developed using the MCNP code. The model was used to determine a calibration factor between the reals rate and the mass of the sample. Measurements with a calibrated ${ }^{252} \mathrm{Cf}$ source were used to verify the model. With a Monte Carlo based approach the mass of the mixed oxide pellets is within a few percent from the nominal values, except for strongly asymmetrical configurations where the deviation is up to about $20 \%$. The results reveal the importance of an accurate background correction and of accounting for surrounding materials of the building such as walls, floor and ceiling in the Monte Carlo model.

Keywords —Neutron coincidence counting; reference nuclear material; waste characterization.

\section{INTRODUCTION}

7 he EC-funded CHANCE project (Characterization of 1 Conditioned Nuclear Waste for its Safe Disposal in Europe) aims to establish an understanding of current characterization methods and quality control schemes for conditioned radioactive waste in Europe. Furthermore, the CHANCE project develops, tests and validates already identified and novel nondestructive assay (NDA) techniques, to improve the radiological characterization of conditioned radioactive waste by reducing the uncertainty on the inventory of radionuclides. One of the innovative technologies for conditioned radioactive waste packages containing high alpha activities and relatively low beta/gamma activities is calorimetry. Currently, an experimental programme is being run to demonstrate in which cases calorimetry combined with various NDA techniques such as gamma-ray spectrometry and passive neutron coincidence counting could reduce the global uncertainty on the inventory of radionuclides. Within this program, three different 200 L mock-up drums containing certified reference nuclear material and one $200 \mathrm{~L}$ drum filled with real unconditioned decommissioning waste, are measured. Once all results are available, the different uncertainty sources for each individual technique will be quantified, followed by a combination of the three techniques using a Bayesian approach. This paper exclusively reports on the neutron coincidence counting measurement results of the three mock-up drums containing reference material. The use of neutron coincidence counting for waste characterization is also investigated in the MICADO project [1].

In this paper we will first outline how we apply neutron coincidence counting to determine the mass of material in the assayed drums. We will then present the measurement campaign by describing the used detectors and electronics, the measured drums and sources; hereafter we describe the Monte Carlo model; finally, we will discuss the obtained results in terms of masses and compare them with the nominal ones.

The uncertainties in this paper are quoted at a $68 \%$ 
confidence limit. We quote the uncertainties between parentheses as recommended in paragraph 7.2.2 of Ref. [2].

\section{NeUtron COINCIDENCE COUNTING}

In general, radioactive materials mainly emit neutrons according to three processes: $(\alpha, n)$ reactions, spontaneous fissions, and neutron-induced fissions. No difference is shown in a neutron detector when neutrons emitted from the different processes are detected. However, $(\alpha, n)$ reactions always emit only one neutron per event, whereas more than one neutron may be emitted in case of fission events. Neutron coincidence counting uses this difference to estimate the quantity of material undergoing spontaneous fission in the sample being measured [3].

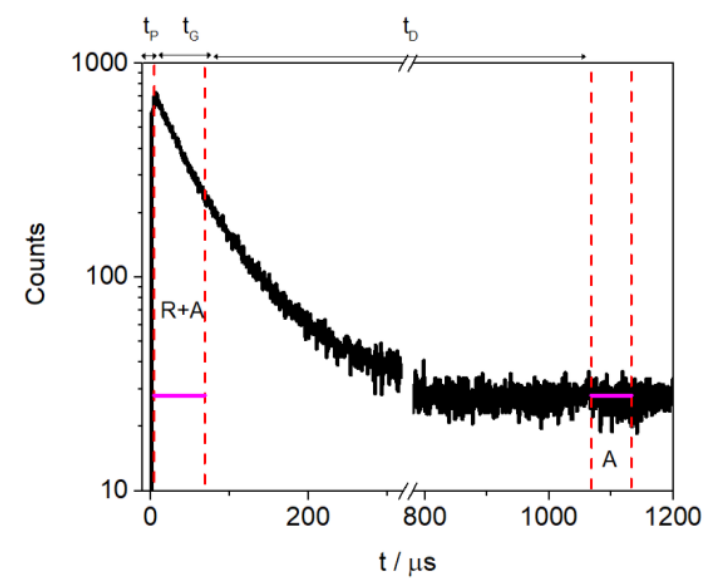

Figure 1: Rossi-alpha distribution from the measurement of a ${ }^{252} \mathrm{Cf}$ source.

Figure 1 shows the Rossi-Alpha distribution (RAD) for a ${ }^{252} \mathrm{Cf}$ source, which represents the time distribution of neutron detections after an arbitrary neutron detection.

If the conditions of the point model are satisfied [4], the Rossi-alpha distribution can be described by the sum of an exponential term and a constant. The constant term is related to so-called accidental time-uncorrelated events and the exponential term is related to the so-called real coincident events. The exponential term is characterized by a die-away time $(\tau)$ which is the mean neutron lifetime in the detector and is determined mainly by the detector geometry. The area of the exponential term represent the total number of coincident events.

In a neutron coincidence measurements, one typically does not measure the RAD over the whole time range but rather in two time intervals (gates) that are open after the detection of each event. Right after the detection of the arbitrary neutron event (at time $\mathrm{t}=0 \mathrm{~s}$ ) a short time interval, also called pre-delay $t_{P}$, is taken to account for the effects of electronic dead-times. After the pre-delay, a gate $t_{G}$ is taken to count the $R+A$ events, followed by a long delay $t_{D}$, and by a second gate to count the $A$ events. The gates for the calculation of the $R+A$ and $A$ events have the same duration. It is common practice to choose a predelay in the order of a few $\mu$ s (e.g. $4.5 \mu$ s), the gate width between 32 and $64 \mu \mathrm{s}$, and the long delay around $1000 \mu \mathrm{s}$ [5]. The determination of the quantity of material undergoing spontaneous fission from the observables $R+A$ and $A$ is explained in Sections IV and V.

\section{EXPERIMENTS}

\section{A. Slab counters and electronics}

Two Canberra WM3400 slab counters [6] were used for the measurements within the CHANCE project. A picture of the detectors during the measurement of a drum is shown in Figure 2 .

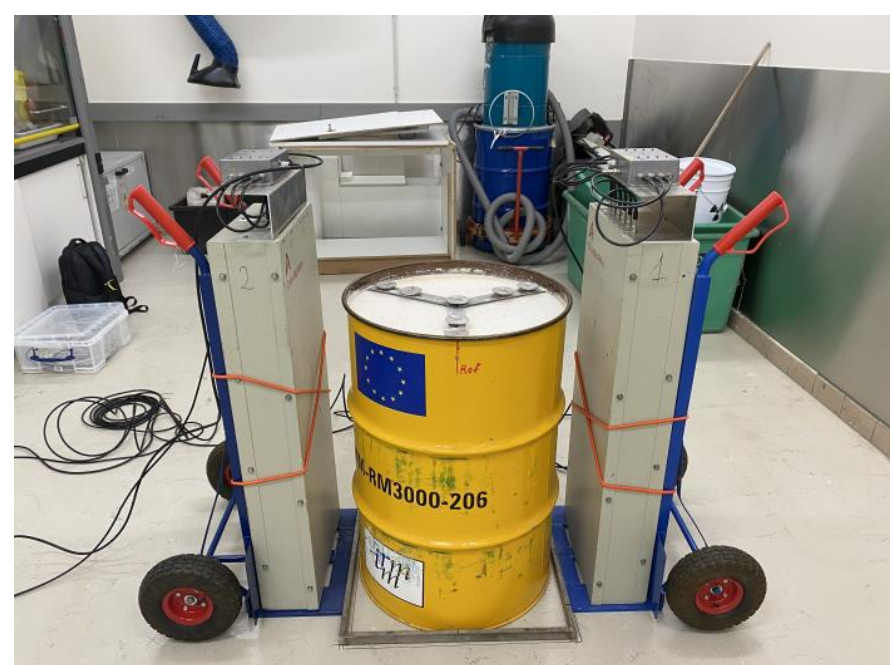

Figure 2: Picture of a measurement with the WM3400 slab counters and the ESARDA drum 206.

Each WM3400 counter measures $104 \mathrm{~cm}$ (height) $\times 41 \mathrm{~cm}$ (width) $\times 16 \mathrm{~cm}$ (depth) and has an approximate weight of 50 $\mathrm{kg}$. To facilitate the movement and positioning of the WM3400 each slab counter has been fixed to a moving cart as shown in Figure 2. The WM3400 counter contains six ${ }^{3} \mathrm{He}$ tubes (active length of $91.44 \mathrm{~cm}$ and diameter of $2.54 \mathrm{~cm}$ ) which are positioned in-line and embedded in a polyethylene slab. The polyethylene slab is then surrounded by $1-\mathrm{mm} \mathrm{Cd}$ sheet that covers five sides of the slab. During a measurement the WM3400 should be placed such that the side without the Cd cover is facing the sample being measured. An additional aluminum sheet covers the slab counter and provides further protection to the inner components.

The default electronic system of the WM3400 has been replaced to reduce the electronic noise and gamma-ray sensitivity [7]. The updated electronic system includes a dedicated preamp for each ${ }^{3} \mathrm{He}$ tube, the possibility to adjust for gain differences between the ${ }^{3} \mathrm{He}$ tubes, and an optimised shaping time for ${ }^{3} \mathrm{He}$ detectors. During the CHANCE measurements two types of data acquisition systems were used in parallel. First a system based on JSR-12 shift registers [8] was used. One JSR-12 shift register was connected to each slab counter, and one JSR-12 was used to process the logical OR of the signals coming from both slab counters. In addition, the time stamps of the neutron detections were recorded with the MCA-527 multi-channel analyzer equipped with time stamp processing firmware [9]. The shift registers recorded the measurement time $t$, the Total events $T$, Real+Accidental events $R+A$, and Accidental events $A$. The time stamps recorded by the 
MCA-527 were further processed off-line with a dedicated software to determine $T, R+A$, and $A$ events, the multiplicity distribution, as well as to remove system instabilities due to high voltage and fluctuations or interferences.

\section{B. ESARDA drums}

The European Safeguards Research and Development Association (ESARDA) constructed a set of 16 so-called reference waste drums (also called ESARDA drums) [10]. Due to the high-level of design information and certification the ESARDA drums were conceived for intercomparison exercises and calibration of NDA equipment devoted to the characterization of plutonium-bearing waste drums.

Three ESARDA drums with the following filling materials were selected for the measurements within the CHANCE project:

- ESARDA drum 201: homogeneous filling with ethafoam

- ESARDA drum 205: heterogeneous filling with ethafoam, PVC, and steel

- ESARDA drum 206: central section with extruded polystyrene and outer layer of mortar

ESARDA drums 201 and 205 were used in their original state, while drum 206 was modified according to the characteristics mentioned above. A central section with extruded polystyrene with a density of $37 \mathrm{~kg} / \mathrm{m}^{3}$ was added to ESARDA drum 206 to limit its total weight to about $300 \mathrm{~kg}$.

The ESARDA drums were designed with 7 vertical channels where sources can be inserted. A schematic representation of these channels is shown in Fig. 3 and Fig. 4.

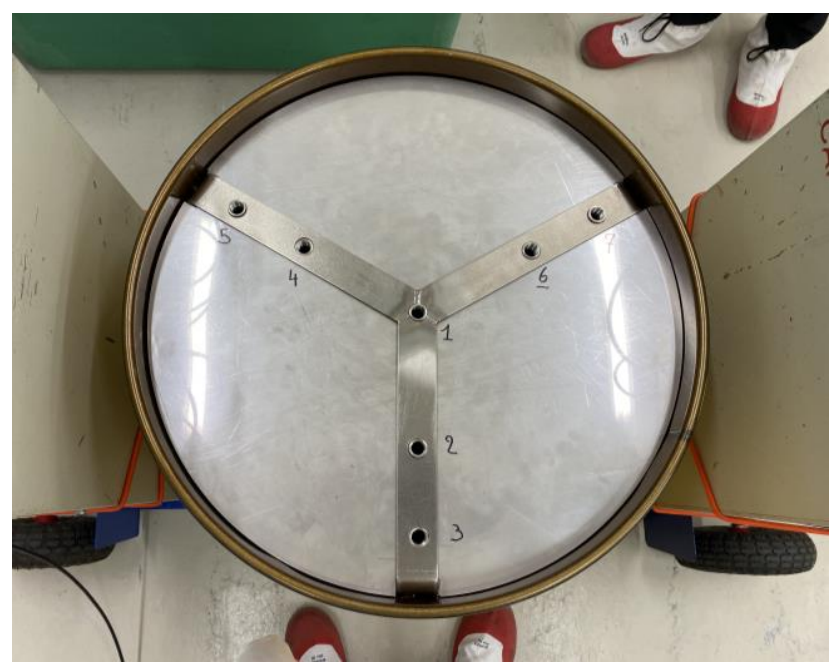

Figure 3: Top view from an ESARDA drum showing the locations of the vertical channels for the positioning of radioactive sources.

To ensure an accurate positioning of the drum and detectors a square metal frame, was placed around the drum. The two slab counters were positioned in contact with the metal frame on opposite sides. By using the metal frame the distance between the slab counters was either 74 or $76 \mathrm{~cm}$ in the two different measurement campaigns. The detectors had to be repositioned manually and the positioning of the slab counters could be reproduced with an accuracy of less than $1 \mathrm{~cm}$. In addition, we verified that the distance between the detectors at the top and at the bottom varied, depending on the measurement point, within $1 \mathrm{~cm}$. The metal frame can be seen in Figure 2 at the bottom of the ESARDA drum.

\section{ESARDA sources}

Two sets of $\mathrm{PuO}_{2}$ sources [11] were used during the CHANCE measurements:

- $\mathrm{C} 80180: 1$ pin with approximately $10.05 \mathrm{~g} \mathrm{PuO}_{2}$ in a single point

- $\mathrm{C} 10100: 7$ pins, each with approximately $1.5 \mathrm{~g} \mathrm{PuO}_{2}$ distributed over three axial points

\section{TABLE I: ISOTOPIC COMPOSITION OF THE PU SOURCES (REFERENCE DATE: END OF 1996).}

\begin{tabular}{rr}
\hline \hline Pu isotope & Mass fractionition \\
\hline${ }^{238} \mathrm{Pu}$ & $1.32 \%$ \\
${ }^{239} \mathrm{Pu}$ & $64.45 \%$ \\
${ }^{240} \mathrm{Pu}$ & $23.83 \%$ \\
${ }^{241} \mathrm{Pu}$ & $5.75 \%$ \\
${ }^{242} \mathrm{Pu}$ & $4.60 \%$ \\
${ }^{241} \mathrm{Am}$ & $4.10 \%$
\end{tabular}

The mass fractions for different radionuclides of the $\mathrm{Pu}$ sources is included in Table I [12].

Given the structure of the ESARDA drums the pin with $\sim 10.05 \mathrm{~g}$ of $\mathrm{PuO}_{2}$ was placed in several positions to assess the sensitivity of the measurement technique to the source location. The second set of $\mathrm{Pu}$ sources was used to simulate the condition of a distributed source throughout the drum volume as shown in Fig. 4.

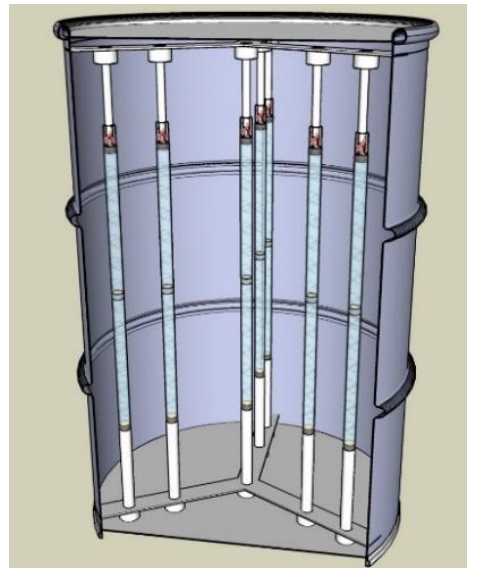

Figure 4: Positioning of the radioactive sources for the 7 pins configuration in a 3D model of a 200 L mock up drum.

In addition to the $\mathrm{Pu}$ sources, a ${ }^{252} \mathrm{Cf}$ source with a nominal activity of $37 \mathrm{kBq}$ on 15 August 2015 was used also as calibration source. The ${ }^{252} \mathrm{Cf}$ source was used in the standard measurement setup without the presence of a drum or inserted in one of the ESARDA drums. 


\section{Considered measurements}

The list of measurements carried out with the slab counters within the CHANCE project is summarized in Table II. The measurement campaign took place in two periods, March 2020 and March 2021, due to an interruption related to the Covid-19 outbreak. For the first two measurements in Table II, no drum was present in the room and the sources (when present) were placed between the detectors. Equal distancing between source and detector was ensured with the metal frame shown in Fig. 2.

The measurements with the ESARDA drum 201 consisted in a background measurement with only the drum placed between the detectors, measurements with the ${ }^{252} \mathrm{Cf}$ source or $\mathrm{C} 80180$ source placed in different positions within the drum, and measurement with the $\mathrm{C} 10100$ sources placed in all available positions. A similar series of measurements were conducted with the ESARDA drum 206, but in this case a total of 7 measurements were carried out with the C80180 source considering all measurement positions except for position 4 . The measurement in position 3 was carried out twice to assess the repeatability of the measurement approach. The measurements with the ESARDA drum 205 consisted in the measurement of background, ${ }^{252} \mathrm{Cf}$ source, and the $\mathrm{C} 10100$ sources.

\section{TABLE II}

Background subtracted totals and reals rate values for the measured combinations of drums and sources at different positions. The uncertainty on the totals rate was always at most $0.1 \mathrm{~s}^{-1}$.

\begin{tabular}{|c|c|c|c|c|}
\hline Drum & Source & Position & $T_{\text {exp }} / \mathrm{s}^{-1}$ & $R_{\text {exp }, m} / \mathrm{s}^{-1}$ \\
\hline \multirow[t]{2}{*}{ None } & ${ }^{252} \mathrm{Cf}$ & 1 & 27.1 & $0.77(<0.01)$ \\
\hline & C80180 & 1 & 242.3 & $1.75(0.01)$ \\
\hline \multirow[t]{9}{*}{201} & ${ }^{252} \mathrm{Cf}$ & 1 & 29.8 & $0.76(<0.01)$ \\
\hline & & 3 & 25.9 & $0.43(<0.01)$ \\
\hline & & 7 & 47.7 & $1.58(0.01)$ \\
\hline & C80180 & 1 & 262.3 & $1.54(0.01)$ \\
\hline & & 2 & 226.7 & $1.12(0.01)$ \\
\hline & & 3 & 176.4 & $0.67(0.01)$ \\
\hline & & 5 & 333.0 & $2.72(0.02)$ \\
\hline & & 7 & 320.8 & $2.54(0.02)$ \\
\hline & C10100 & All & 260.5 & $1.53(0.01)$ \\
\hline \multirow[t]{2}{*}{205} & ${ }^{252} \mathrm{Cf}$ & 1 & 26.0 & $0.64(<0.01)$ \\
\hline & C10100 & All & 239.7 & $1.39(0.01)$ \\
\hline \multirow[t]{10}{*}{206} & ${ }^{252} \mathrm{Cf}$ & 1 & 24.5 & $0.50(<0.01)$ \\
\hline & & 7 & 35.1 & $1.07(0.01)$ \\
\hline & C80180 & 1 & 218.9 & $0.99(0.01)$ \\
\hline & & 2 & 182.0 & $0.66(0.01)$ \\
\hline & & 3 & 91.1 & $0.14(>0.01)$ \\
\hline & & 3 & 90.9 & $0.14(<0.01)$ \\
\hline & & 5 & 240.8 & $1.19(0.01)$ \\
\hline & & 6 & 341.6 & $2.66(0.02)$ \\
\hline & & 7 & 358.8 & $2.93(0.02)$ \\
\hline & C10100 & All & 222.6 & $1.08(0.01)$ \\
\hline
\end{tabular}

The measurement data from the time stamps recorded with the MCA527 were analyzed by means of Python [13] scripts, in the Spyder environment version 4 [14] . The scripts first carried out stability checks of the data by monitoring the total count rate over 10 seconds interval. Then the data were analyzed to determine the totals and the reals rate for each measurement. The background subtraction was then carried out and the net total and net real count rates, indicated with $T_{\text {exp }}$ and $R_{\text {exp } m}$ respectively, were determined.

The obtained data were used to determine the mass of the assayed sample by applying two methodologies, one based on the point model approximation and one based on Monte Carlo modeling.

\section{POINT MODEL APPROACH}

\section{A. Theory}

Starting from basic principles, theories were developed $[15,16,4]$ that express the observables of neutron coincidence or multiplicity measurement as function of the mass of neutron emitting radionuclides and other parameters. This model, called the point model, can be applied in case the assayed sample has point geometry, no neutrons return from the detector to the sample, the time response function of the moderator detector assembly is a pure exponential function with decay constant, and there are no dead time losses of signals. Other conditions also apply but we refer to the reference for the full description.

In absence of multiplication, the theoretical estimates from the point model of Hage [4] are the following for the totals and reals rate:

$$
T_{p m}=\varepsilon F_{s} M v_{s(1)}[1+\alpha]
$$

$$
R_{p m}=\varepsilon^{2} F_{S} M^{2} v_{s(2)}
$$

Where

- $\varepsilon$ is the (total) detection efficiency

- $F_{s}$ the spontaneous fission rate of the test item

- $\alpha=\frac{F_{S}}{S_{\alpha}}$

- $\mathrm{S}_{\alpha}$ the $(\alpha, \mathrm{n})$ neutron emission rate of the test item

- $v_{j(\mu)}=\sum_{\mu=v}^{\infty}\left(\begin{array}{l}\mu \\ v\end{array}\right) P_{j \mu} \quad$ is the $\mu_{\mathrm{th}}$ factorial moment of the neutron emission distribution $P$ by spontaneous fission

The experimental observables from the recorded pulse train can be obtained by looking at the signal triggered multiplets. In our case we limit to multiplets of order 0 and 1 and the experimental observables are the total count rate $T_{\text {exp }}$ and the measured reals rate $R_{\text {exp }, m}$ :

$$
R_{\text {exp }}=\frac{(R+A)-A}{t f}=\frac{R_{\text {exp }, m}}{f}
$$


Where

$$
f=\int_{t_{p}}^{t_{p}+t_{g}} \frac{e^{-t / \tau}}{\tau} d t=e^{-t_{p} / \tau}\left(1-e^{-t_{g} / \tau}\right)
$$

and $t$ is the measurement time.

The $f$ factor accounts for the finite duration of the trigger gate and the presence of a predelay. The given expression for $f$ holds only for an exponential RAD.

\section{B. Data Analysis}

In this work we applied the model equation (1) and (2) using the measured observables $T_{\text {exp }}$ and $R_{\text {exp }, m}$ to derive the detection efficiency $\varepsilon$ and the spontaneous fission rate $F_{s}$. The term $\alpha$ as well as the factorial moments of the neutron emission distribution associated to the assayed item were derived from the radionuclide composition of the sample. The $f$ factor was obtained from the average of three values determined from the RAD: one from fitting the RAD with 2 exponentials, one from the observed RAD and correction for predelay assuming a 1 exponential function, one from the observed RAD and correction for predelay assuming a 2 exponentials function.

From the $F_{s}$ one can determine the mass of the sample by dividing by $F_{s}$, the number of spontaneous fission per second and per gram.

$$
m_{c}=\frac{F_{s}}{F_{s}^{\prime}}
$$

\section{MONTE CARLO APPROACH}

\section{A. Description of the MCNP model}

The Monte Carlo code MCNP6 [17] was used to carry out a full scale modelling of the measurement setup. The model included the detectors and the used source(s) and drum, as well as the room containing the measurement setup (walls, ceiling and floor).

Since the specifications of detectors, drums and sources were very well known, the geometry and material composition could be modelled with a high degree of fidelity. A cross section of an example of a modeled geometry is shown in Fig. 5.

MCNP6 was run in the so-called analog mode i.e. tracking event-by-event the interactions of neutrons rather than their average behaviors. A tally of the type F8 with the CAP special treatment [18] was used. Such a treatment allows to record neutron absorption in given radionuclides and cells in given time intervals after a neutron absorption has taken place at time zero. The neutron multiplicity is scored in user defined intervals. It was found that neglecting walls, ceiling and floor resulted in an underestimation between $7 \%$ and $28 \%$ on the simulated reals rate. The simulations only account for neutrons emitted by the spontaneous fission and do not account for reals induced by $(\alpha, n)$ reaction which are present only in the presence of a multiplying sample. For the $\mathrm{Cf}$ source the spontaneous fission of ${ }^{252} \mathrm{Cf}$ was considered while for the $\mathrm{PuO}_{2}$ ESARDA sources we considered ${ }^{240} \mathrm{Pu}$.

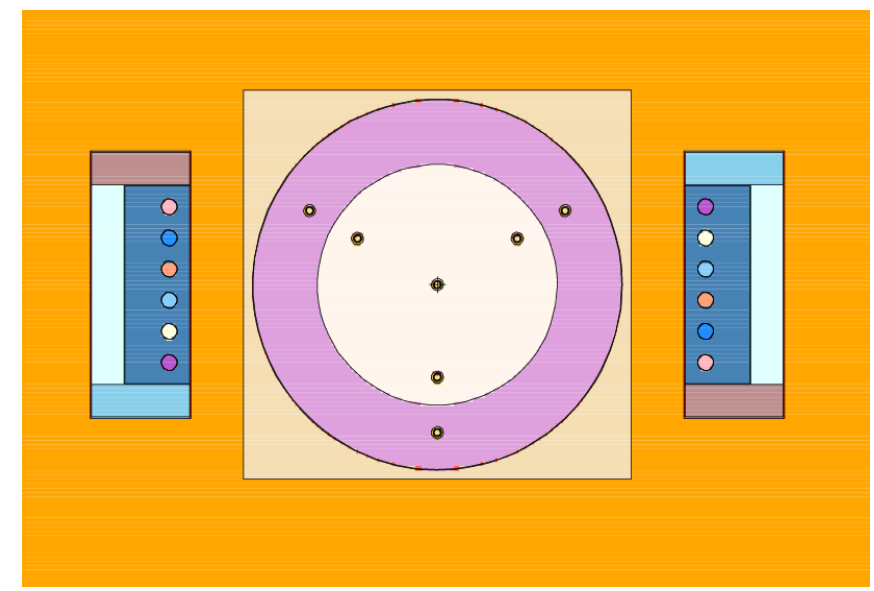

Figure 5: Horizontal cross section of the Monte Carlo model of the measurement setup for the 206 drum and the slab counters.

\section{B. Post processing}

The neutron multiplicity due to spontaneous fission were simulated in two time intervals both of duration of $64 \mu \mathrm{s}$ : the first one called $R+A$ gate and starting after $4.5 \mu$ s from the spontaneous fission event; the second one called $A$ gate and starting $1,000 \mu \mathrm{s}$ after the end of $R+A$ gate. From the multiplicity we determined the reals $R_{\text {sim, } m}$ per spontaneous fission event as:

$$
R_{\text {sim }, m}=\frac{S \times\left(m_{R+A(1)}-m_{A(1)}\right)}{N_{F}}
$$

Where:

- $\quad S$ is the total count rate

- $\quad N_{F}$ is the number of fission events in the MCNP simulation

$\begin{array}{ll}-\quad & m_{R+A(k)}=\sum_{x=k}^{\infty}\left(\begin{array}{l}x \\ k\end{array}\right) n_{x} \\ - & m_{A(k)}=\sum^{\infty}\left(\begin{array}{l}x \\ k\end{array}\right) b_{x}\end{array}$

- $\quad m_{A(k)}=\sum_{x=k}^{\infty}\left(\begin{array}{l}x \\ k\end{array}\right) b_{x}$

- $\quad n_{x}$ is the normalised multiplicity distribution in the $\mathrm{R}+\mathrm{A}$ gate.

- $\quad b_{x}$ is the normalised multiplicity distribution in the $\mathrm{A}$ gate.

- $\left(\begin{array}{l}x \\ k\end{array}\right)=\frac{x !}{(x-k) ! k !}$ is the binomial coefficient

Note that the reals rate $R_{\text {sim, } m}$ was calculated with the predelay and trigger gate used in the measurement to determine $R_{\text {exp } m}$ and therefore a correction for the $f$ factor defined in equation 3 is not needed.

\section{Data Analysis}

From the reals per fission event $R_{\text {sim, }}$ obtained from the MCNP6 simulations, the number of ${ }^{240} \mathrm{Pu}$ fission per second was determined. By using the spontaneous fission rate of ${ }^{240} \mathrm{Pu}$ $F^{\prime}{ }_{s, 240 P u}$ we then determined the mass of ${ }^{240} \mathrm{Pu}$-equivalent. 


$$
m_{{ }^{440}{ }_{P u_{e q}}}=\frac{\frac{R_{\text {exp }, m}}{R_{\text {sim }, m}}}{F_{s, 240 P u}^{\prime}}
$$

The mass of ${ }^{240} \mathrm{Pu}$-equivalent, $m^{240} \mathrm{Pu}_{e q}$, is the mass of ${ }^{240} \mathrm{Pu}$ that results in the same number of reals rate, $R_{\text {exp }, m}$.

The estimate mass $m_{c}$ was then determined by [19]:

$$
m_{c}=\frac{m^{240}{ } u_{e q}}{w}
$$

where

$$
w=\sum_{i} \frac{Q_{i}}{Q_{240}^{24 u}} N_{i}
$$

with

$$
Q=F_{s}^{\prime} v_{s(2)}=\lambda \beta_{s f, i} \frac{N_{a v}}{A} v_{s(2)}
$$

- $\quad F_{s}^{\prime}$ the spontaneous fission rate per gram

- $\quad \lambda$ the decay constant

- $\quad \beta_{s f}$ the branching ratio for spontaneous fission

- $\quad N_{a v}$ is the Avogadro constant

- $\quad A$ is the atomic mass

- $\quad v_{s(2)}$ is the second factorial moment of the neutron emission distribution by spontaneous fission

- $\quad N_{i}$ mass fraction of the considered radionuclide

The factorial moment of the neutron emission distribution by spontaneous fission were taken from a compilation of different sources e.g. [20,21,22].

In this work, we did not simulate the neutron emitted by $(\alpha, n)$ reactions. In absence of multiplication, $(\alpha, n)$ neutrons are time uncorrelated and therefore their impact is limited to the term of order zero in the multiplicity distributions. Such a term cancels out when calculating the reals rate.

The impact of accounting for other radionuclides present in the sources such as ${ }^{250} \mathrm{Cf}$ in the $\mathrm{Cf}$ source, or other even $\mathrm{Pu}$ isotopes in the $\mathrm{PuO}_{2}$ sources was studied. Due to the different emission spectrum and multiplicity distributions the calculated $R_{s i m, m}$ may slightly change and hence the calculated mass $m_{c}$. The maximum observed deviation in the calculated mass $m_{c}$ was $3.3 \%$ for the bare ${ }^{252} \mathrm{Cf}$ source.

\section{RESULTS AND CONCLUSIONS}

The obtained masses $m_{c}$ with the previously outlined procedures were compared with the nominal masses $m_{n}$ of the measured samples. The obtained ratios $m_{c} / m_{n}$ for the considered cases are given in Table III for the point model and Monte Carlo approaches. The quoted uncertainties result from count statistics on the net totals rate $T_{\text {exp }}$, the net reals rate $R_{\text {exp }, m}$ and on the reals per spontaneous fission event $R_{\text {sim }, m}$ from Monte
Carlo simulations, and have been obtained by first-order Taylor expansion-based error propagation.

The masses obtained by applying the point model are in good agreement with the nominal ones for the ${ }^{252} \mathrm{Cf}$ measurements both in the bare configuration and for the measurement with a drum. For the measurements with the ESARDA sources we observe a systematic overestimation of the mass. The reason of such a difference could be related to the fact for the ${ }^{252} \mathrm{Cf}$ the $(\alpha, n)$ contribution is negligible, when compared to the spontaneous fission term, while the contribution from $(\alpha, n)$ is significant for the $\mathrm{PuO}_{2}$ sources and directly impacts the total count rate as indicated in Eq. 1. Given the open measurement geometry, the measured total count rate is also affected by neutron scattering in the surroundings and should be properly accounted for to correctly meet the point model conditions.

\begin{tabular}{|c|c|c|c|c|}
\hline Drum & Source & Position & $\mathrm{m}_{\mathrm{c}} / \mathrm{m}_{\mathrm{n}}$ & $\mathrm{m}_{\mathrm{c}} / \mathrm{m}_{\mathrm{n}}$ \\
\hline & & & Point Model & Monte Carlo \\
\hline \multirow[t]{2}{*}{ None } & ${ }^{252} \mathrm{Cf}$ & 1 & $0.95(0.01)$ & $1.00(0.01)$ \\
\hline & C80180 & 1 & $1.21(0.03)$ & $1.05(0.01)$ \\
\hline \multirow[t]{9}{*}{201} & ${ }^{252} \mathrm{Cf}$ & 1 & $1.03(0.01)$ & $0.98(0.01)$ \\
\hline & & 3 & $1.08(0.02)$ & $0.98(0.01)$ \\
\hline & & 7 & $1.04(0.01)$ & $0.86(<0.01)$ \\
\hline & C80180 & 1 & $1.38(0.03)$ & $0.98(0.01)$ \\
\hline & & 2 & $1.46(0.05)$ & $0.99(0.01)$ \\
\hline & & 3 & $1.50(0.07)$ & $1.01(0.01)$ \\
\hline & & 5 & $1.36(0.03)$ & $0.96(0.01)$ \\
\hline & & 7 & $1.37(0.03)$ & $0.90(0.01)$ \\
\hline & C10100 & All & $1.41(0.04)$ & $1.00(0.01)$ \\
\hline \multirow[t]{2}{*}{205} & ${ }^{252} \mathrm{Cf}$ & 1 & $0.99(0.01)$ & $0.97(0.01)$ \\
\hline & C10100 & All & $1.31(0.03)$ & $0.98(0.01)$ \\
\hline \multirow[t]{10}{*}{206} & ${ }^{252} \mathrm{Cf}$ & 1 & $1.01(0.01)$ & $0.99(0.01)$ \\
\hline & & 7 & $1.01(0.01)$ & $0.88(<0.01)$ \\
\hline & C80180 & 1 & $1.42(0.04)$ & $1.02(0.01)$ \\
\hline & & 2 & $1.49(0.05)$ & $1.03(0.01)$ \\
\hline & & 3 & $1.60(0.09)$ & $1.07(0.02)$ \\
\hline & & 3 & $1.56(0.08)$ & $1.04(0.02)$ \\
\hline & & 5 & $1.45(0.04)$ & $1.01(0.01)$ \\
\hline & & 6 & $1.41(0.03)$ & $1.14(0.01)$ \\
\hline & & 7 & $1.38(0.03)$ & $1.26(0.01)$ \\
\hline & C10100 & All & $1.37(0.04)$ & $1.00(0.01)$ \\
\hline
\end{tabular}

TABLE III

$m_{c} / m_{n}$ values for the measured combinations of drums and sources at different positions

For the masses obtained by applying the Monte Carlo approach, the overall agreement between the obtained mass and nominal mass is very good. However, we observe that for asymmetrical measurement positions, such as position 7 , deviations up to $26 \%$ are observed. Such a deviation is present both for the ${ }^{252} \mathrm{Cf}$ and the $\mathrm{C} 80180$ source although the sign is not always consistent: the C80180 mass is overestimated in 
position 7 in drum 206 and underestimated in drum 201, while the ${ }^{252} \mathrm{Cf}$ mass is always underestimated. The reason of these deviations is not yet understood. While in symmetrical measurements the contributions to the total and reals count rate from the two detectors are essentially the same, in an asymmetrical configuration one detector can contribute much more to the reals rate as, in the point model approximation, the reals rate is proportional to the square of the neutron detection efficiency [4]. Therefore the accurate position of the detector and source is even more important in an asymmetrical configuration.

Future work will focus on integrating these results with similar studies, based on the same sources and drums, for gamma spectrometry and calorimetry, where uncertainty quantification will be performed using state-of-the-art Bayesian probabilistic modelling. Additionally, application to a real unconditioned waste drum, with unknown radionuclide inventory, is also envisioned, to test the methodology on a case more representative of daily practice.

\section{ACKNOWLEDEMENTS}

This project has received funding from the Euratom research and training programme 2014-2018 under grant agreement No 755371.

\section{REFERENCES}

[1] Available online: https://www.micado-project.eu/. Last accessed 28/04/2021.

[2] Evaluation of measurement data - Guide to the expression of uncertainty in measurement, JCGM 100:2008, First edition September 2008, available https://www.iso.org/sites/JCGM/GUM/JCGM100/C045315ehtml/C045315e.html?csnumber=50461

[3] Ensslin E., 1991. "Principles of Neutron Coincidence Counting" in Passive Nondestructive Assay of Nuclear Materials, NuREG/CR-5550, LA-UR-90-732.

[4] D.M. Cifarelli \& W. Hage Nucl. Instr. \& Meth. A251 (1986) 550-563

[5] Menlove H. O., 1991. "Neutron coincidence instruments and applications". in Passive Nondestructive Assay of Nuclear Materials, NuREG/CR-5550, LA-UR-90-732.

[6] https://www.mirion.com/products/wm3400-passive-neutron-slabcounter. Last accessed 28/04/2021

[7] Boogers E., et al., 2019. "Improved electronics for 3He based neutron counters". Proceedings of the 2019 ANIMMA conference.

[8] Canberra, 2006. "Model JSR-12 Neutron Coincidence Analyzer User's Manual", Canberra Industries Inc.

[9] GBS, 2021. https://www.gbselektronik.de/media/download_gallery/mca527_neutron.pdf. Last accessed 28/04/2021

[10] Bignan G. et al., 1995. "Proposal of an intercomparison exercise for the characterization of waste containing plutonium by NDA methods." Working document of the $17^{\text {th }}$ annual ESARDA meeting.

[11] Bignan G., et al., 1996. "Preparation of standard drums for the calibration of nuclear waste measuring facilities. Final report". CEA note technique NT-SSAE-LSMR 98/0016.

[12] A. Verbruggen, R. Garcia-Galan, Certification of BC1413, GE/R/SIM/7/98 June 1998, IRMM Stable Isotope Measurement Unit

[13] https://www.python.org/]

[14] https://docs.spyder-ide.org ]

[15] R. Dierckx \& W. Hage (1983) Neutron Signal Multiplet Analysis for the Mass Determination of Spontaneous Fission Isotopes, Nuclear Science and Engineering, 85:4, 325-338,

[16] W. Hage \& D.M. Cifarelli Nucl. Sci \& Eng. 89 (1985) 159-176

[17] MCNP6 Users Manual - Code Version 6.1.1 beta, LA-CP-14-00745, June 2014.
[18] M. T. Swinhoe, J. S. Hendricks, and D. R. Mayo, "MCNPX for Neutron Multiplicity Detector Simulation," Los Alamos National Laboratory report LA-UR-04-8025 (2004).

[19] Report D8.2 "Identification of the sources of uncertainty related to individual methods", 2020, MICADO, H2020 NFPR 10 -2018, \#847641

[20] Safeguards and Fissile Materials Management, a Preliminary procedure for the treatment of time correlation analyser measurement data for nondestructive assay of P, W. Hage K. Caruso, Technical Note I.07.C3.86.115, 1987

[21] Norman E. Holden \& Martin S. Zucker (1986) Prompt neutron multiplicities for the transplutonium nuclides, Radiation Effects: Incorporating Plasma Science and Plasma Technology, 96:1-4, 289-292, DOI: $10.1080 / 00337578608211755$

[22] Langner, D G, Stewart, J E, Pickrell, M M, Krick, M S, Ensslin, N, and Harker, W C. Sun . "Application Guide to Neutron Multiplicity Counting". United States. https://doi.org/10.2172/1679. https://www.osti.gov/servlets/purl/1679. 